\title{
SYNTHESIS AND COMPOSITION CHARACTERIZATION OF $\mathrm{K}_{0.5} \mathrm{Na}_{0.5} \mathrm{NbO}_{3}-\mathrm{KSr}_{2} \mathrm{Nb}_{5} \mathrm{O}_{15}$ CERAMICS
}

\author{
"LIANGLIANG LIU, ZHUANGZHUANG GUO, YONGQIANG WANG, HAIWEI JIANG
}

College of Materials Science and Engineering, Taiyuan University of Technology, Taiyuan 030024, PR China

"E-mail: liuliangliang_2004@163.com

Submitted April 2, 2018; accepted April 24, 2018

\begin{abstract}
Keywords: $\mathrm{KSr}_{2} \mathrm{Nb}_{5} \mathrm{O}_{15}$, Ferroelectric ceramics, Microstructure, Dielectric property
$\mathrm{KSr}_{2} \mathrm{Nb}_{5} \mathrm{O}_{15}$ modified potassium sodium niobate ceramics $((1-x) \mathrm{KNN}-x \mathrm{KSN})$ were fabricated by a pressure-less solid-state method, using Bi as a sintering aid. Synthesis and composition characterization of the $(1-x) K N N-x K S N$ ceramics were investigated. It was found that the KSN addition was favorable to the densification of the $(1-x) K N N-x K S N$ ceramics due to the improvement of sintering temperature and the absence of abnormal grain growth. Induced by KSN, the KNN phase structure spontaneously transformed from perovskite to tetragonal tungsten bronze. The result suggested that for preparing $K N N$-based ceramics with high performance it is necessary to control the phase transformation. This study also opens up a new avenue for the investigation of the relationship between structure and performance in KNN ceramics.
\end{abstract}

\section{INTRODUCTION}

The attention on piezoelectric ceramics has been moving to $\mathrm{Pb}$-free materials owing to increasing environmental and health concerns. Among $\mathrm{Pb}$-free materials, $\left(\mathrm{K}_{0.5} \mathrm{Na}_{0.5}\right) \mathrm{NbO}_{3}(\mathrm{KNN})$-based ceramics have been widely studied recently due to their high Curie temperature and good electrical properties $[1,2]$. It is well known that pure KNN ceramics prepared by traditional sintering always lack sufficient density and high dielectric loss [3]. Various second phases have been used to prepare dense KNN-based ceramics with excellent performance $[1,2]$. For example, an extremely high piezoelectric coefficient $d_{33}$ of $570 \mathrm{pC} / \mathrm{N}$ was obtained by optimizing the composition [4]. Recently, Du's groups attained a large recoverable energy storage density of $4.08 \mathrm{~J} \cdot \mathrm{cm}^{-3}$ by the formation of $\left(\mathrm{K}_{0.5} \mathrm{Na}_{0.5}\right) \mathrm{NbO}_{3}-\mathrm{Bi}\left(\mathrm{Me}_{2 / 3} \mathrm{Nb}_{1 / 3}\right) \mathrm{O}_{3}$ solid solutions (where $\mathrm{Me}^{2+}=\mathrm{Mg}$ and $\mathrm{Zn}$ ) [5]. Yang's group observed high transparency in $\mathrm{K}_{0.5} \mathrm{Na}_{0.5} \mathrm{NbO}_{3}-$ $-\mathrm{Sr}\left(\mathrm{Zn}_{1 / 3} \mathrm{Nb}_{2 / 3}\right) \mathrm{O}_{3}$ ceramics synthesized by conventional pressureless sintering [6]. Obviously, these reports provided a guideline on future research directions for the development of KNN ceramics. An effective method to improve the performance of $\mathrm{KNN}$ ceramics consist in by doping with a second phase.

Our recent study suggests that using of $\mathrm{KSr}_{2} \mathrm{Nb}_{5} \mathrm{O}_{15}$ (KSN) particles prepared by a molten salt method promoted densification of ceramics during sintering $[7,8]$. According to the literature, doping $\mathrm{KNN}$ with a small amount of $\mathrm{Sr}$ improved the density and electric properties of KNN [9, 10]. Marija et al [11] reported that a good transparency could be obtained due to the decrease of grain size in $\mathrm{KNN}-\mathrm{SrTiO}_{3}$ ceramics with increasing amounts of $\mathrm{Sr}$. However, the composition cha- racteristics of $\mathrm{K}_{0.5} \mathrm{Na}_{0.5} \mathrm{NbO}_{3}-\mathrm{KSr}_{2} \mathrm{Nb}_{5} \mathrm{O}_{15}$ (KNN-KSN) ceramics have not yet been investigated so far. In this work, $\mathrm{KSr}_{2} \mathrm{Nb}_{5} \mathrm{O}_{15}$ was used as a second phase. $\mathrm{KNN}-$ $-\mathrm{KSN}$ ceramics were prepared by traditional sintering technology. Synthesis and composition characteristics of $\mathrm{KNN}-\mathrm{KSN}$ ceramics were investigated.

\section{EXPERIMENTAL}

In the present study $(1-x) \mathrm{K}_{0.5} \mathrm{Na}_{0.5} \mathrm{NbO}_{3}-x \mathrm{KSr}_{2} \mathrm{Nb}_{5} \mathrm{O}_{15}$ $((1-x) \mathrm{KNN}-x \mathrm{KSN})(x=0,0.15,0.30$ and 0.45$)$ ceramics were prepared by traditional solid-state reaction methods. KNN powder was fabricated by reactive sintering of $\mathrm{K}_{2} \mathrm{CO}_{3}(99 \%), \mathrm{Na}_{2} \mathrm{CO}_{3}(99 \%)$ and $\mathrm{Nb}_{2} \mathrm{O}_{5}(99.6 \%)$. These reagents were provided by Sinopharm Chemical Reagent Co., Ltd. (China). The raw materials were mixed homogeneously by ball milling in ethanol for $10 \mathrm{~h}$ using zirconia balls, then calcined in a temperature of $900^{\circ} \mathrm{C}$ for $4 \mathrm{~h}$. The rates of heating and cooling were $5^{\circ} \mathrm{C} \mathrm{min}^{-1}$ and $2^{\circ} \mathrm{C} \mathrm{min}{ }^{-1}$, respectively. KSN powder was prepared by a molten salt synthesis (MSS) method. Details of the synthesis of the KSN particles have been reported previously [12]. KNN, KSN powder and excess 2 mol. \% $\mathrm{Bi}_{2} \mathrm{O}_{3}(99.9 \%)$ were mixed by ball milling for $24 \mathrm{~h}$ with ethanol. After drying, the mixture was granulated with a binder of 6 wt. \% poly vinyl alcohol (PVA) and then pressed into disk-shaped pellets with diameter $12 \mathrm{~mm}$ and thickness $1.3 \mathrm{~mm}$. After burning off the PVA, all disk samples were sintered at $1050 \sim 1250^{\circ} \mathrm{C}$ or $2 \mathrm{~h}$ with a constant heating rate of $5^{\circ} \mathrm{C} \cdot \mathrm{min}^{-1}$ and finally furnacecooling to room temperature with a rate of $2^{\circ} \mathrm{C} \cdot \mathrm{min}^{-1}$. Silver electrodes were coated on both surfaces of each sintered samples by firing at $600^{\circ} \mathrm{C}$ for $10 \mathrm{~min}$. 
Bulk densities of the sintered samples were determined by using the Archimedes method. The relative density was calculated based on the theoretical densities of KSN $\left(5.002 \mathrm{~g} \cdot \mathrm{cm}^{-3}\right)$ and KNN $\left(4.51 \mathrm{~g} \cdot \mathrm{cm}^{-3}\right)$. The phase structure of the KSN powder crystals and ceramics was characterized by X-ray diffraction (XRD, Panalytical X'Pert PRO, The Netherlands) using $\mathrm{Cu} \mathrm{K} \alpha$ radiation and a graphite monochromator. Microstructures of the polished and thermally-etched disks were identified by scanning electron microscopy (SEM, Quanta 600 FEG, USA). The curves of the dielectric constant $(\varepsilon)$ and dielectric loss $(\tan \delta)$ against temperatures between $30^{\circ} \mathrm{C}$ to $500^{\circ} \mathrm{C}$ at $10 \mathrm{kHz}, 100 \mathrm{kHz}$ and $1 \mathrm{MHz}$ were performed with an LCR precision electric bridge (Hewlett-Packard HP4284, Palo Alto, USA).

\section{RESULTS AND DISCUSSION}

Figure 1 shows the photographs of the $(1-x) \mathrm{KNN}-$ $-x \mathrm{KSN}$ ceramics $(x=0,0.15,0.30$ and 0.45$)$ prepared by sintering at temperatures in the range from 1050 to $1250^{\circ} \mathrm{C}$. As shown in the figure, samples sintered at $1050^{\circ} \mathrm{C}$ did not exhibit any significant shrinkage. The KNN (without KSN) sample appeared bubbling phenomenon during heating up $1150^{\circ} \mathrm{C}$ and the large shrinkage occurred for $0.85 \mathrm{KNN}-0.15 \mathrm{KSN}$ sample. Heating to $1200^{\circ} \mathrm{C}$ resulted in further shrinkage for $0.7 \mathrm{KNN}-0.3 \mathrm{KSN}$ and $0.55 \mathrm{KNN}-0.45 \mathrm{KSN}$ samples. When the sintering temperature approached $1250^{\circ} \mathrm{C}$, all samples showed overburning characteristics, such as bubbling and cracking. Obviously, the optimum sintering temperature for these samples was in the range from $1050^{\circ} \mathrm{C}$ to $1225^{\circ} \mathrm{C}$. The temperature was increased with the increase of KSN content. Table 1 shows the density of the $(1-x) \mathrm{KNN}-x \mathrm{KSN}$ ceramics prepared at the optimized sintering temperature. The relative densities of the $(1-x) \mathrm{KNN}-x \mathrm{KSN}$ ceramics were greater than $90 \%$. The density increased and then decreased as a function of the KSN content, and reached a maximum value at $x=0.15$. During the sintering stage, KSN grains consumed the surrounding smaller KNN grains and grew competitively. A small amount of KSN promoted the ion diffusion due to the existence of vacancy defects $[7,8,12]$. Increasing KSN content casued that the large KSN grains impinged on one another and had less small grains to consume. This may be the reason for the occurrence of the optimal density in the $0.85 \mathrm{KNN}-0.15 \mathrm{KSN}$ ceramics. Thus, samples sintered at $1050^{\circ} \mathrm{C}, 1150^{\circ} \mathrm{C}, 1200^{\circ} \mathrm{C}$ and $1225^{\circ} \mathrm{C}$ for $\mathrm{KNN}-0 \mathrm{KSN}, 0.85 \mathrm{KNN}-0.15 \mathrm{KSN}$, $0.70 \mathrm{KNN}-0.30 \mathrm{KSN}$ and $0.55 \mathrm{KNN}-0.45 \mathrm{KSN}$, respectively, were used to examine the phase structure, microstructure and dielectric property.

Table 1 . Density of the $(1-x) \mathrm{KNN}-x \mathrm{KSN}$ ceramics prepared at the optimized sintering temperature.

\begin{tabular}{ccccc}
\hline$x$ & $\begin{array}{c}\text { Sintering } \\
\text { temperature } \\
\left({ }^{\circ} \mathrm{C}\right)\end{array}$ & $\begin{array}{c}\text { Density } \\
\left(\mathrm{g} \cdot \mathrm{cm}^{-3}\right)\end{array}$ & $\begin{array}{c}\text { Theoretical } \\
\text { density } \\
\left(\mathrm{g} \cdot \mathrm{cm}^{-3}\right)\end{array}$ & $\begin{array}{c}\text { Relative } \\
\text { density } \\
(\%)\end{array}$ \\
\hline 0 & 1050 & 4.245 & 4.51 & 94.12 \\
0.15 & 1150 & 4.506 & 4.584 & 98.31 \\
0.30 & 1200 & 4.397 & 4.658 & 94.41 \\
0.45 & 1225 & 4.526 & 4.731 & 95.66 \\
\hline
\end{tabular}

Figure 2 displays the XRD patterns of the $(1-x)$ $\mathrm{KNN}-x \mathrm{KSN}$ ceramics $(x=0,0.15,0.30$ and 0.45$)$. A single phase was obtained for the sample in the case of $x=0$. When $\mathrm{x} \geq 0.15$, a secondary phase was detected. The extra peaks could be indexed to the $\mathrm{KSr}_{2} \mathrm{Nb}_{5} \mathrm{O}_{15}$ (JCPDF 34-0123) phase that exhibits tetragonal tungstenbronze (TTB) type structure. An amplified zoom of the XRD profiles in the $2 \theta$ range from $31^{\circ}$ to $33^{\circ}$ is shown in the inset of Figure 2. It can be seen that the diffraction lines shifted toward larger $2 \theta$ angle with increasing KSN content (the position of the strongest peak of KSN being $\left.32.136^{\circ}\right)$. However, the peak did not exhibit splitting. This behavior could be related with the formation of a solid solution.

Figure 3 shows the evolution of the intensity ratio of KSN characteristic (410) peak to the strongest peak as a function of the KSN content. The value of this ratio significantly increased, suggesting that the addition of

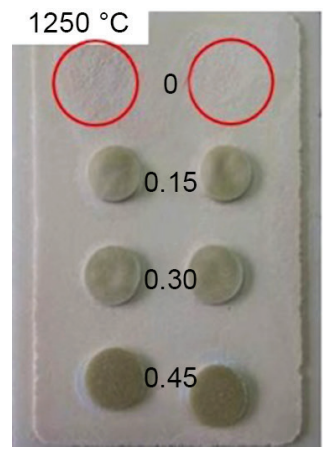

a)

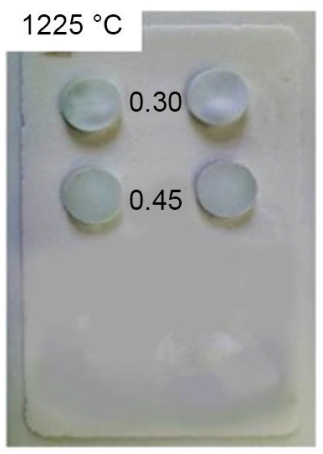

b)

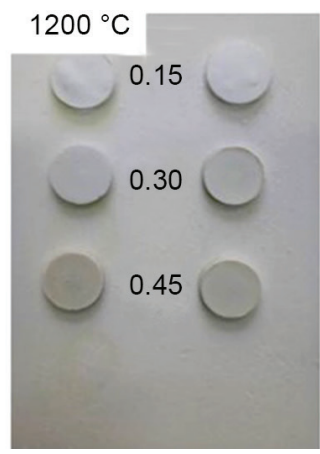

c)

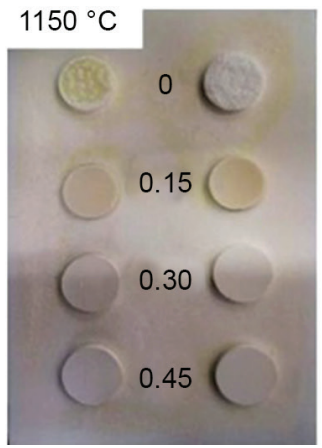

d)

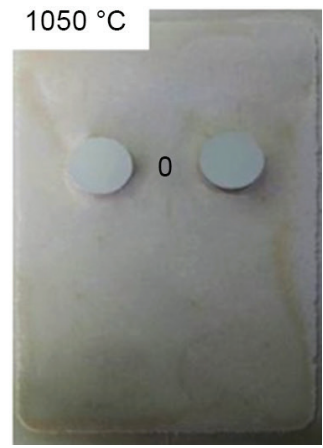

e)

Figure 1. Photographs of the $(1-x) \mathrm{KNN}-x \mathrm{KSN}$ ceramics $(x=0,0.15,0.30$ and 0.45$)$. 
Liu L., Guo Z., Wang Y., Jiang H.

KSN modified the crystalline structure. With the increase of KSN content, the crystal structure gradually changed from perovskite to TTB structure. In KNN ceramics, TTB secondary phase was usually formed owing to the volatilization of the potassium and sodium ions [1, 2]. The KNN phase also could be transformed easily from
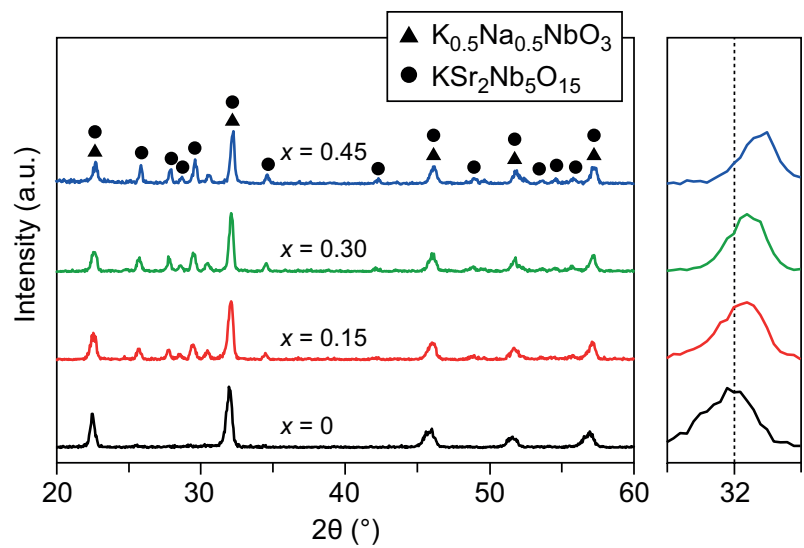

Figure 2. XRD patterns of the $(1-x) \mathrm{KNN}-x \mathrm{KSN}$ ceramics $(x=0,0.15,0.30$ and 0.45$)$.

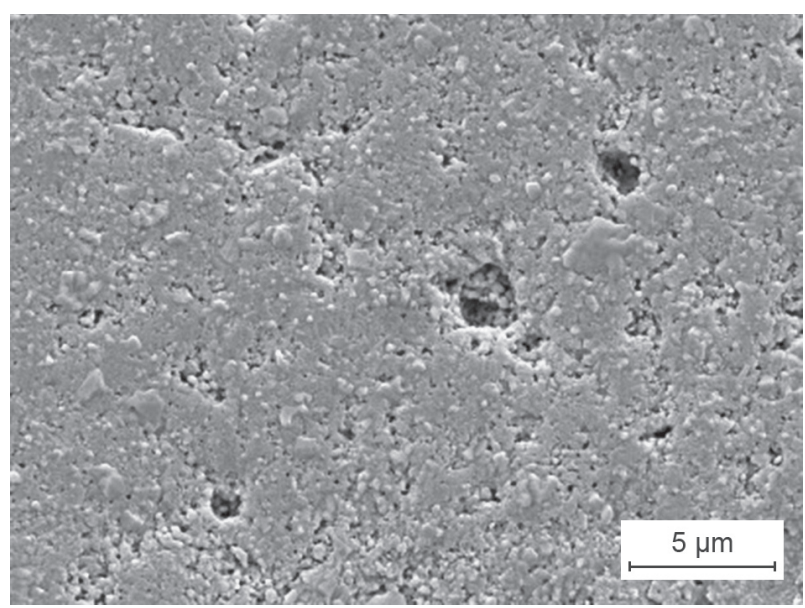

a) $x=0$

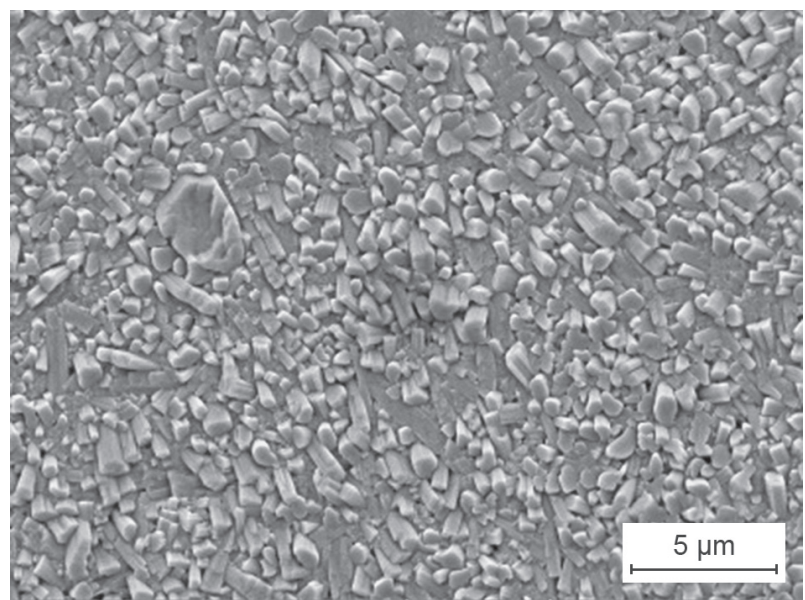

c) $x=0.30$ the TTB phase [13]. Therefore, our results suggested that $\mathrm{K}^{+}$and $\mathrm{Na}^{+}$had diffused into the KSN lattice to form a solid solution in the $(1-x) \mathrm{KNN}-x \mathrm{KSN}$ ceramics.

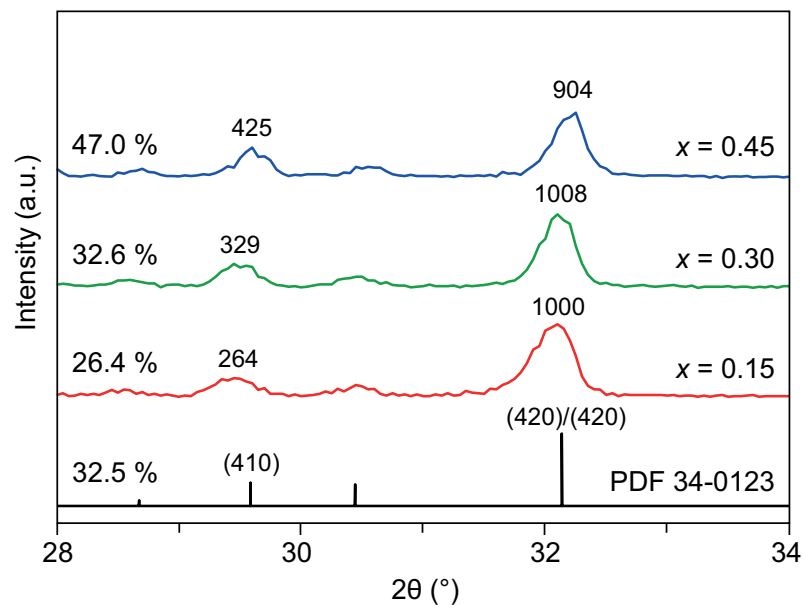

Figure 3. Magnified XRD patterns in the $28^{\circ}-34^{\circ}$ region of the $(1-x) \mathrm{KNN}-x \mathrm{KSN}$ ceramics.

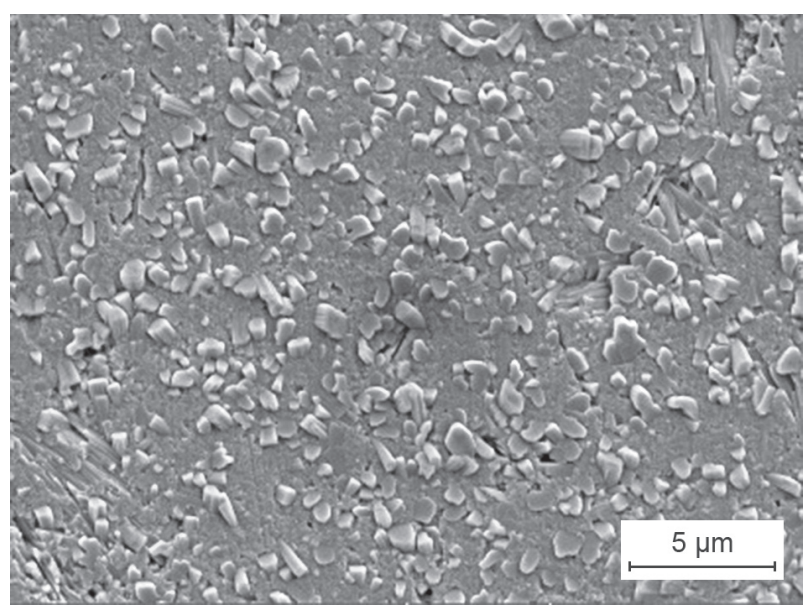

b) $x=0.15$

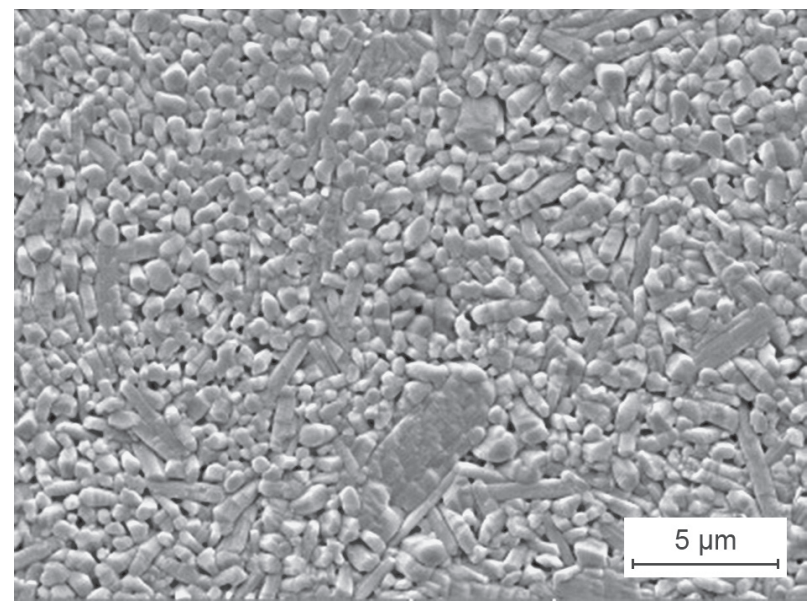

d) $x=0.45$

Figure 4. Microstructure of the $(1-x) \operatorname{KNN}-x \operatorname{KSN}$ ceramics: a) $x=0, \mathrm{~b}) x=0.15$, c) $x=0.30$ and d) $x=0.45$. 
The SEM images of the $(1-x) \mathrm{KNN}-x \mathrm{KSN}$ ceramics are shown in Figure 4. As seen in Figure 4a, the typical microstructures of the alkaline niobate ceramics are small and cubic equiaxed grains. A change of the microstructure was observed with KSN content as displayed in Figures $4 \mathrm{~b} \sim \mathrm{d}$. As the KSN content increased, the percentage of columnar grain increased significantly. Furthermore, in the case of $x=0.45$ the cube-like grains disappeared and only columnar grains remained. It was found that the columnar grain size was uniform for the case of $x=0.45$ and almost the same with that of $x=0.15$ and $x=0.3$ (seen in Figures $4 \mathrm{~b}$ and $\mathrm{c}$ ) although the sintering temperature was the highest. Abnormal grain growth did not occur, contrary to what is often observed for other KNN-based ceramics $[1,2]$. In other words, KSN particles inhibited the KNN grain growth. In addition, the porosity first decreased and then increased with increasing KSN content. This result suggested that a small amount of KSN particles promoted the density of KNN ceramics.

The assessment of phase structure needs more accurate measurement methods, and so the temperature dependence of dielectric constant was used to further confirm the phase structure. Figure 5 shows the temperature dependence of the dielectric constant $(\varepsilon)$ and loss

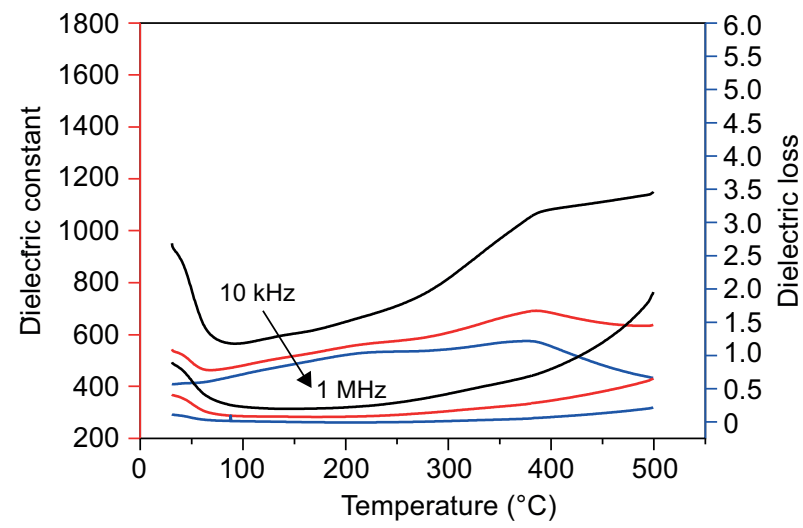

a) $x=0$

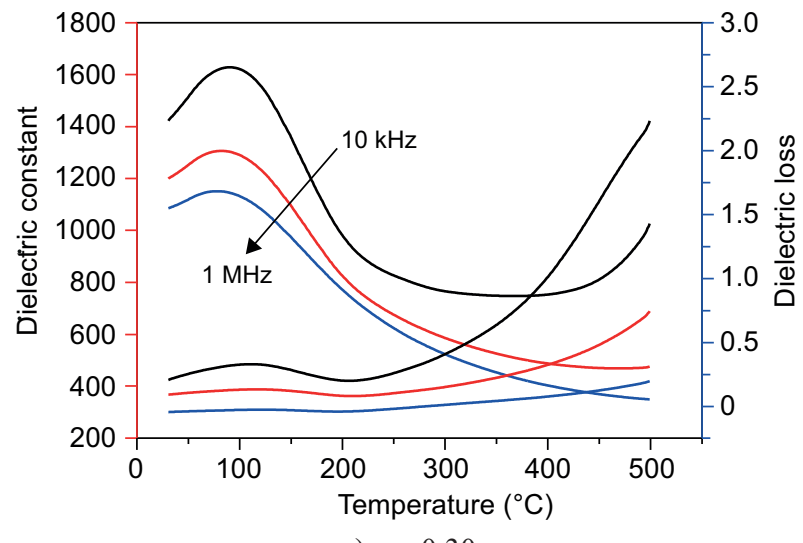

c) $x=0.30$ $(\tan \delta)$ of the $(1-x) \mathrm{KNN}-x \mathrm{KSN}$ ceramics at different measuring frequencies $(10 \mathrm{kHz}, 100 \mathrm{kHz}$ and $1 \mathrm{MHz})$. High dielectric losses were found for all samples over a wide temperature range $\left(30^{\circ} \mathrm{C}\right.$ to $\left.500^{\circ} \mathrm{C}\right)$. This can be attributed to space charge effects due to doping with $\mathrm{Bi}_{2} \mathrm{O}_{3}$.

In order to investigate the effect of the KSN content, the measured frequency of $1 \mathrm{MHz}$ was chosen to remove space charge polarization contributions as shown in Figure 6. In the case of $x=0$, the curve shows two anomalies. The anomaly at $\sim 200^{\circ} \mathrm{C}$ was explained by the orthorhombic-tetragonal $\left(T_{\mathrm{O}-\mathrm{T}}\right)$ phase transition, as well as a dielectric peak at $\sim 400^{\circ} \mathrm{C}$ for the $T_{\mathrm{C}}$ (tetragonalcubic phase transition). By comparison with that of pure $\mathrm{KNN}$ ceramics $[1,2]$, the broadening of the peaks suggests a diffuse phase transition. That is, the ceramics transformed gradually a "normal" to a diffuse phase transition when doping with $\mathrm{Bi}^{3+}$. Similar phenomena have appeared in $\mathrm{Bi}^{3+}$-doped $\mathrm{KSN}$ ceramics $[7,8]$. This behavior could be attributed to an increase in the level of structural disorder due to the introduction of $\mathrm{Bi}^{3+}$ into the KNN structure and the subsequent occurrence of cationic vacancies. As seen in Figure 6, it is obvious that the addition of KSN decreased $T_{\mathrm{C}}$, and $T_{\mathrm{O} \text { - } \mathrm{T}}$ disappeared $T_{\mathrm{C}}$ of the $(1-x) \mathrm{KNN}-x \mathrm{KSN}$ ceramics decreased gradual-

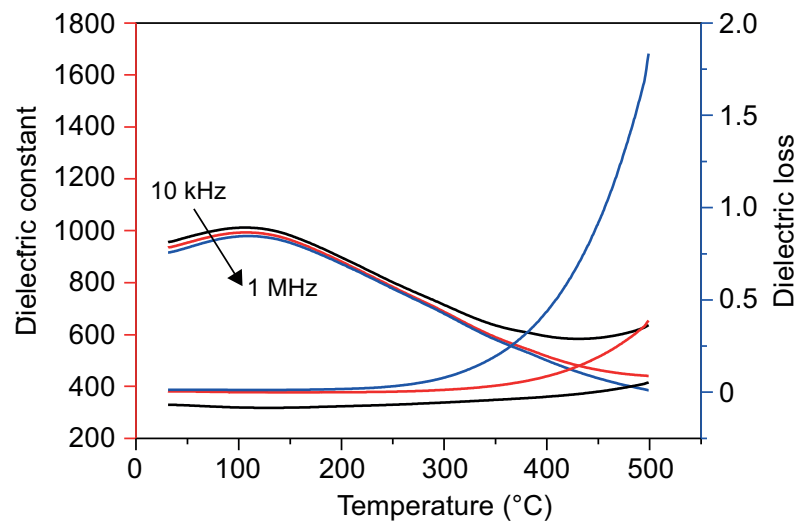

b) $x=0.15$

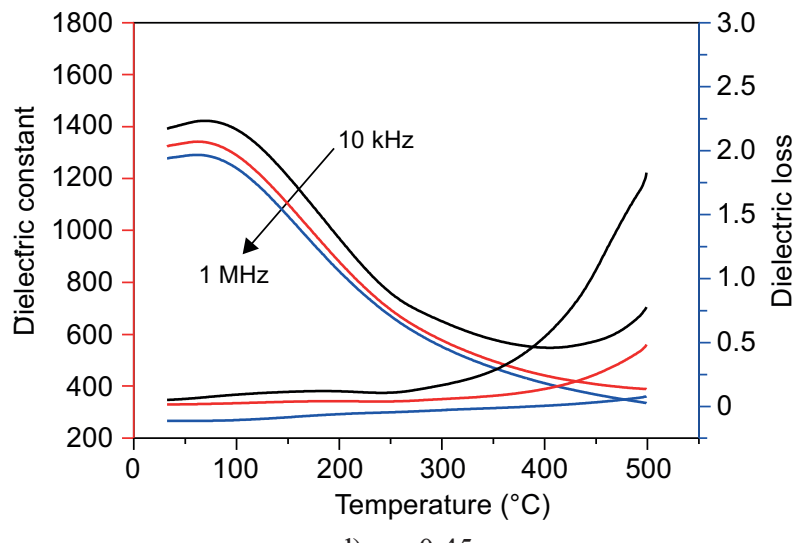

d) $x=0.45$

Figure 5. Temperature dependence of dielectric constant $(\varepsilon)$ of the $(1-x) \operatorname{KNN}-x \operatorname{KSN}$ ceramics: a) $x=0, \mathrm{~b}) x=0.15$, c) $x=0.30$ and d) $x=0.45$. 
ly with increasing KSN content. It should be noted that their $T_{\mathrm{C}}$ values were consistent with that of KSN ceramics. These results further confirmed the formation of a solid solution when adding with KSN into KNN. Generally, the dielectric properties are affected by the point defect concentration, especially oxygen vacancy, which is commonly found in electronic materials $[14,15]$. As is well known, oxygen vacancies are involved in KNN ceramics owing to the volatilization of the alkali metal ions ( $\mathrm{K}$ and $\mathrm{Na}$ ) during high-temperature processing. So, more work needs to be done for understanding the influence of point defects, which will promote the development of excellent electrical properties in the KNN ceramics.

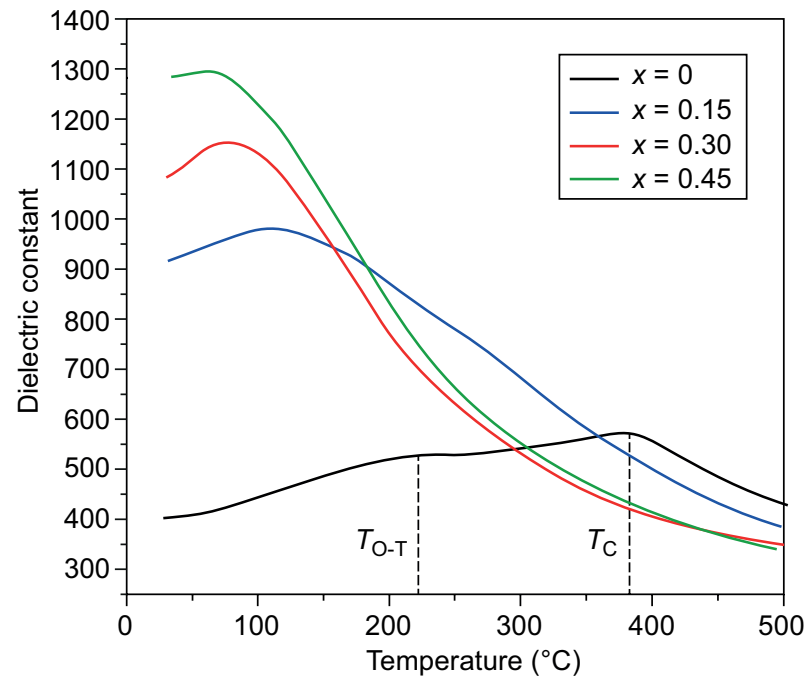

Figure 6. Temperature dependence of dielectric constant $(\varepsilon)$ of the $(1-x) \mathrm{KNN}-x \mathrm{KSN}$ ceramics at $1 \mathrm{MHz}$.

\section{CONCLUSIONS AND OUTLOOK}

In this work, $(1-x) \mathrm{KNN}-x \mathrm{KSN}$ ceramics were designed to study the effect of KSN content on their sintering behavior and composition characteristics. The results showed that with the increase of KSN content, the sintering temperature of the $(1-x) \mathrm{KNN}-x \mathrm{KSN}$ ceramics increased and no abnormal grain growth (AGG) phenomenon was observed. This will give a guide to prepare dense KNN ceramics with uniform grain size by the conventional solid-state reaction method. It should be noted that a solid solution was formed due to the diffusion of $\mathrm{K}^{+}$and $\mathrm{Na}^{+}$into the KSN lattice. That is, the formation of TTB phase was promoted with the addition of KSN. This feature will be investigated in more detail in the future in order to obtain a better understanding of the relationship between structure and performance in KNN ceramics.

\section{Acknowledgments}

This work was supported by the National Natural Science Foundation of China (No. 51502191).

\section{REFERENCES}

1. Li J.F., Wang K., Zhu F.Y., Cheng L.Q., Yao F.Z. (2013): $(\mathrm{K}, \mathrm{Na}) \mathrm{NbO}_{3}$-based lead-free piezoceramics: fundamental aspects, processing technologies, and remaining challenges. Journal of the American Ceramic Society, 96, 3677-3696. doi:10.1111/jace.12715

2. Zheng T., Wu J., Xiao D., Zhu J., Wang X., Lou X. (2015): Composition-driven phase boundary and piezoelectricity in potassium-sodium niobate-based ceramics. ASC Applied Materials Interfaces, 7, 20332-20341. doi: 10.1021/acsami. $5 \mathrm{~b} 06033$

3. Su Y.L., Chen X.M., Yu Z.D., Lian H.L., Zheng D.D., Peng J.H. (2017): Comparative study on microstructure and electrical properties of $\left(\mathrm{K}_{0.5} \mathrm{Na}_{0.5}\right) \mathrm{NbO}_{3}$ lead-free ceramics prepared via two different sintering methods. Journal of Materials Science, 52, 2934-2943. doi:10.1007/s1085

4. Xu K., Li J., Lv X., Wu J., Zhang X., Xiao D., Zhu J. (2016): Superior piezoelectric properties in potassium-sodium niobate lead-free ceramics. Advanced Materials, 28, 8519-8523. doi:10.1002/adma.201601859

5. Shao T., Du H., Ma H., Qu S., Wang J., Wang J., Wei X., Xu Z. (2017): Potassium-sodium niobate based lead-free ceramics: novel electrical energy storage materials. Journal of Materials Chemistry A, 5, 554-563. doi: 10.1039/C6TA07803F

6. Chai Q., Yang D., Zhao X., Chao X., Yang Z. (2017): Lead-free $(\mathrm{K}, \mathrm{Na}) \mathrm{NbO}_{3}$-based ceramics with high optical transparency and large energy storage ability. Journal of the American Ceramic Society, doi: 10.1111/jace.15392.

7. Liu L., Gao F., Zhang Y., Sun H. (2014): Dense $\mathrm{KSr}_{2} \mathrm{Nb}_{5} \mathrm{O}_{15}$ ceramics with uniform grain size prepared by molten salt synthesis. Journal of Alloys and Compounds, 616, 293-299. doi: 10.1016/j.jallcom.2014.07.155

8. Liu L., Gao F., Hu G., Liu J., Li J. (2013): Microstructure and electrical properties of potassium strontium niobate $\left(\mathrm{KSr}_{2} \mathrm{Nb}_{5} \mathrm{O}_{15}\right)$ ceramics. Journal of Alloys and Compounds, 580, 93-100. doi: 10.1016/j.jallcom.2013.05.093

9. Malic B., Bernard J., Holc J., Kosec M. (2005): Strontium doped $\mathrm{K}_{0.5} \mathrm{Na}_{0.5} \mathrm{NbO}_{3}$ based piezoceramics. Ferroelectrics, 314, 149-156. doi:10.1080/00150190590926373

10. Hrescak J., Drazic G., Deluca M., Arcon I., Kodre A., Dapiaggi M., Rojac T., Malic B., Bencan A. (2017): Donor doping of $\mathrm{K}_{0.5} \mathrm{Na}_{0.5} \mathrm{NbO}_{3}$ ceramics with strontium and its implications to grain size, phase composition and crystal structure. Journal of the European Ceramic Society, 37, 2073-2082. doi: 10.1016/j. jeurceramsoc.2016.12.053

11. Marija K., Bobnar V., Hrovat M., Bernard J., Malic B., Holc J. (2004): New lead-free relaxors based on the $\mathrm{K}_{0.5} \mathrm{Na}_{0.5} \mathrm{NbO}_{3}$ $-\mathrm{SrTiO}_{3}$ solid solution. Journal of Materials Research, 19, 1849-1854. doi: 10.1557/JMR.2004.0229

12. Liu L., Gao F., Hu G., Liu J., Li J. (2013): Effect of excess $\mathrm{Nb}_{2} \mathrm{O}_{5}$ on the growth behavior of $\mathrm{KSr}_{2} \mathrm{Nb}_{5} \mathrm{O}_{15}$ particles by molten salt synthesis. Powder Technology, 235, 806-813. doi: 10.1016/j.powtec.2012.11.040

13. Luo L., Chen C., Luo H. (2015): The effects of precursors on the morphology and microstructure of potassium sodium niobate nanorods synthesized by molten salt synthesis. CrystEngComm, 17, 8710-8719. doi: 10.1039/C5CE01382H

14. Wu J., Xiao D., Zhu J. (2015): Potassium-sodium niobate leadfree piezoelectric materials: past, present, and future of phase boundaries. Chemical Reviews, 115, 2559-2595. doi: 10.1021/ cr5006809

15. Liu Z.Q., Leusink D.P., Wang X., Lü W.M., Gopinadhan K., Annadi A., Zhao Y.L., Huang X.H., Zeng S.W., Huang Z., Srivastava A., Dhar S., Venkatesan T., Ariando (2011): Metalinsulator transition in $\mathrm{SrTiO}_{3-\mathrm{x}}$ thin films induced by frozen-out carriers. Physical Review Letters, 107, 146-802. doi:10.1103/ PhysRevLett.107.146802 